\title{
Diagnosis of Inborn Errors of Metabolism from Blood Spots by Acylcarnitines and Amino Acids Profiling Using Automated Electrospray Tandem Mass Spectrometry
}

MOHAMED S. RASHED, PINAR T. OZAND, MARTIN P. BUCKNALL, AND DOUGLAS LITTLE

Departments of Biological and Medical Research [M.S.R, P.T.O., M.P.B, D.L.] and Pediatrics [P.T.O.],

King Faisal Specialist Hospital and Research Centre, Riyadh 11211, Saudi Arabia

ABSTRACT

\begin{abstract}
Acylcarnitine profiling from blood or plasma samples by electrospray tandem mass spectrometry (ESI-MS/MS) has been rccognized recently as a useful tool in the biochemical diagnosis of propionic acidemia, methylmalonic acidemia together with short-chain and medium-chain acyl-CoA dehydrogenase deficiencies. In the current study, we have investigated the diagnostic capabilities of ESI-MS/MS in other types of organic acidemias and amino acid catabolism disorders. Using multiple scanning functions, we examined the potential for the simultaneous profiling of both acylcarnitines and amino acids, in each of the samples. Our method was found to be specific and accurate; allowing quantification of acylcarnitines and amino acids well below, and significantly above, published normal levels. Complete automation of sample introduction has been achicved, allowing the analysis of up to 200 samples in one injection sequence, at a rate of one sample every $3 \mathrm{~min}$, with excellent separation between successive injections. In our hands, this
\end{abstract}

Acylcarnitine analysis by FAB-MS/MS has been used in the last few years as a rapid and reliable method for the biochemical diagnosis of several inborn errors of metabolism, samples being collected in the form of blood or plasma, spotted onto Guthrie cards (1-3). The soft ionization characteristics of the FAB technique allow the analysis of acylcarnitines, which are charged, polar, and nonvolatile molecules, lacking a strong chromophore. The use of the MS/MS approach eliminates the need for chromatographic separation, thereby shortening the analysis time to 1 or $2 \mathrm{~min}$; after a simple sample preparation procedure. More recently, the MS/MS method has been extended to examine the levels of amino acids. The amino acid profiles, generated in this way have proven useful in the

Received November 14, 1994; accepted May I, 1995.

Corresondence and reprints requests: Dr. Mohamed S. Rashed, King Faisal Specialist Hospital and Research Centre, MBC3, P.O. Box 3354, Riyadh 11211, Saudi Arabia.

Supported by grants provided by Sheikh Rafiq Al Hariri 85-0030 and Research Centre Grant 85-0021. method permits screening for 20 organic acid and amino acid disorders, using a single sample injection. In our laboratory, more than 2000 blood samples have been analyzed, and 52 new cases were diagnosed by this method. We also confirmed the diagnosis of another 75 previously known cases. (Pediatr Res 38: 324-331, 1995)

ESI, electrospray

\section{Abbreviations}

$\mathrm{FAB}$, fast atom bombardment

MS, mass spectrometry

MS/MS, tandem mass spectrometry

GC, gas chromatography

PKU, phenylketonuria

GA, glutaric acidemia

CID, collision-induced dissociation

HMG, hydroxy-3-methylglutaryl-CoA lyase deficiency diagnosis of PKU, maple syrup urine disease, tyrosinemia, and citrullinemia $(2,4)$

This broad spectrum metabolic profiling represents a promising approach for the development of neonatal screening programs for inborn errors of metabolism. Diagnostic FABMS/MS is routinely carried out in the static mode, using manual introduction of individual samples on the tip of a vacuum insertion probe. Attempts to use the continuous-flow FAB to automate sample introduction are still at an experimental stage. Whether static or dynamic FAB is used, it is difficult to maintain the sensitivity of the system which requires frequent cleaning and maintenance.

Recently, the application of ESI-MS/MS was proposed by ourselves and others as a more robust alternative technique to FAB-MS/MS for acylcarnitine profiling in blood $(5,6)$. In a previous study, we outlined the application of ESI-MS/MS in the diagnosis of propionic acidemia, methylmalonic acidemia, and short-chain acyl-CoA dehydrogenase deficiency (6). In the current study, we have applied ESI-MS/MS to the diagnosis of 
several other organic acidemias and amino acid catabolism disorders in which FAB-MS/MS has been shown to be of diagnostic utility. We are also presenting the results of our work on automation of the technique and its application in the quantification of acylcarnitines and amino acids.

\section{METHODS}

Stable isotope standards. Acetyl- $\left[\mathrm{d}_{3}\right.$-methyl $]$ carnitine, octanoyl- $\left[\mathrm{d}_{3}\right.$-methyl $]$ carnitine, and palmitoyl- $\left[\mathrm{d}_{3}-\right.$ methyl $]$ carnitine standards were a generous gift of Dr. David S. Millington (Duke University). Alanine-2,3,3,4- $\mathrm{d}_{4}$, valine- $\mathrm{d}_{8}$, leucine$5,5,5-\mathrm{d}_{3}$, methionine-methyl- $\mathrm{d}_{3}$, phenylalanine-ring- $\mathrm{d}_{5}$, and tyrosine-ring- $\mathrm{d}_{4}$ were purchased from Cambridge Isotope Laboratories (Woburn, MA).

Samples. Blood spots used in this study were collected over a period of about 9 mo from patients referred to the Inborn Errors of Metabolism Unit at the King Faisal Specialist Hospital and Research Centre, Riyadh. Three spots of heparinized blood (75 $\mu \mathrm{L}$ each) were spotted on Schleicher \& Schüll filter paper (S\&S 903; Dassel, Germany), allowed to dry at room temperature for a few hours or overnight, and stored in polypropylene bags at room temperature until analysis.

Unless otherwise noted, the data to be presented here were from new patients that were diagnosed initially by MS/MS analysis of blood before any treatment was carried out. When applicable, the diagnosis was confirmed by urine organic acid and/or plasma amino acid analyses by standard methods, and in some cases by enzyme assays.

Blood sample preparation. Two 3/16-inch circles from each blood spot $(\sim 13 \mu \mathrm{L})$ were punched out, using a standard hole punch, into $1.5-\mathrm{mL}$ polypropylene microcentrifuge tubes (3). The samples were then extracted by shaking for $30 \mathrm{~min}$ in 400 $\mu \mathrm{L}$ of methanol or acetonitrile containing known amounts of the stable isotope-labeled internal standards. The tubes were then centrifuged at $14,000 \mathrm{rpm}$ for $10 \mathrm{~min}$. The supernatants were transferred to $1.1-\mathrm{mL}$ glass vials with conical shaped bottoms (1.1-CTVG vials; obtained from Chromacol Limited; Welwyn Garden City, UK) and evaporated to dryness using a gentle stream of nitrogen. To the residues, $250 \mu \mathrm{L}$ of butanolic $\mathrm{HCl}$ was added (which was prepared by saturating dry $n$ butanol with $\mathrm{HCl}$ gas for $30 \mathrm{~min}$ ). The vials were capped, vortexed, and heated at $65^{\circ} \mathrm{C}$ for $20 \mathrm{~min}$. The resulting mixtures were again dried, and each residue was finally reconstituted in $150 \mu \mathrm{L}$ of acetonitrile/water $(4: 1 \mathrm{vol} / \mathrm{vol})$. The resulting solutions were transferred to $0.3-\mathrm{mL}$ autosampler vials (03-CVG vials; obtained from Chromacol Limited), in preparation for MS/MS analysis.

MS. ESI-MS/MS was carried out on a Fisons Instruments (Altrincham, UK) VG Quattro, triple quadrupole mass spectrometer, equipped with a Jasco model PU-980 HPLC pump and a Jasco model AS-950 autosampler, fitted with a $20-\mu \mathrm{L}$ sample loop (Jasco International Co. Ltd., Tokyo, Japan). Argon was used as the collision gas at a pressure of 2.5 to 3.5 mTorr, and the collision energy was set to $25 \mathrm{eV}$. The ion source temperature was maintained at $70^{\circ} \mathrm{C}$.

For acylcarnitine profiles, precursor ion scans of the peak at $\mathrm{m} / \mathrm{z} 85$ were monitored in the range $\mathrm{m} / \mathrm{z} 250-500$. For amino acid profiling, two neutral loss scan functions were used, one for neutral loss of $102 \mathrm{D}$, and the other for neutral loss of 119 D (4). After a solvent delay time of $0.3 \mathrm{~min}$ from the time of sample injection, the three consecutive scan functions were carried out sequentially using a scan speed of one scan every 2 $\mathrm{s}$ for all functions. Each of the three scan functions was active, in the order listed above, during each of the following time periods (postsolvent delay): 0.0-0.7 $\mathrm{min}, 0.7-1.2 \mathrm{~min}$, and 1.2-1.7 min.

The resolution of MS1 was adjusted in the precursor-ion mode, such that the acylcarnitine peaks were approximately 1.3 $\mathrm{D}$ wide at $15 \%$ height. This gave good sensitivity and allowed the peak detection and centering algorithm to discern peaks separated by only $1 \mathrm{D}$. The resolution of MS2 was set in the daughter-ion mode by observing the daughters of m/z 260 in the range $\mathrm{m} / \mathrm{z} 80-90$. Mass 260 corresponds to the molecular ions $\left(\mathrm{M}^{+}\right)$for both, the butyl ester of acetylcarnitine and the dibutyl ester of glutamic acid. Under CID conditions, the first produces a major fragment at $\mathrm{m} / \mathrm{z} 85$, whereas the latter produces a major fragment at $\mathrm{m} / \mathrm{z} 84$. To minimize any interference from glutamate in the acetylcarnitine signal, the resolution parameters were adjusted to give a valley depth between masses 84 and 85 which was approximately $10 \%$ of the height of mass 84; and to give this resolution linearly throughout the mass range used for all scan functions.

For routine analysis, the data were acquired in the multichannel analysis mode, where all data collected during a particular scan function is recorded as a single summed analog data spectrum. As the individual scan intensities are not recorded, chromatograms cannot be obtained. In this mode, most of the noise is averaged out resulting in a significant improvement in the signal to noise ratio of the spectra. The storage of only summed spectral profiles also dramatically reduces space occupied by each patient's data file on the computer's hard disk.

For development work toward automation, signal intensity chromatograms were needed; therefore the data were acquired using the "MCB" data capture mode. In this mode, the spectral profile data from every scan is recorded, enabling the construction of both mass and total ion chromatograms; thereby allowing examination of interinjection resolution. To obtain spectra of good signal-to-noise quality for postrun analysis, it is essential to sum or average all the individual spectra recorded for each sample. Since only an "averaging" algorithm is currently available, these combined spectra yielded somewhat lower numerical intensities, but nevertheless equal signal-to-noise quality, when compared with those acquired by "summing" in the multichannel analysis mode.

Data processing. A macro was used for processing the MS/MS data. This program, written using the Microsoft Visual Basic program, automates key strokes in the VG Quattro Masslynx software. The operator is required to select the files to be processed by appending their names to the macro's processing table from the sample data directory. For each file, the macro sequentially selects and processes the spectrum corresponding to each scan function. The analog data are smoothed using a Savitzky-Golay algorithm (provided in the Masslynx software), and the smoothed spectrum is printed. The 
macro then centroids and height measures the smoothed data, creating and printing a numerical list of peak positions on the mass scale and their height intensities. This process takes 2.5 min for the three scan functions of each file, using a DECpc 486D2LP personal computer.

Automation of sample introduction. In initial work, manual injections were made using a Rheodyne injector model 7125. In latter work, toward automation of sample introduction, the injector port no. 4 on the autosampler was connected directly via a Rheodyne ferrule to a fused silica tube (VG part no. $6028620 ; 75-\mu \mathrm{m}$ inside diameter; about $65 \mathrm{~cm}$ long). The fused silica tube was connected through a specially drilled double-ended vespel ferrule and a zero dead volume fitting to a 34 gauge stainless steel tube $(0.009$ outside diameter; 0.005 inside diameter) inside the ESI probe. The length of the silica tubing was kept to a minimum to shorten the time it takes for the sample to reach the ion source. This time is termed "solvent delay time" and in our case was about $0.3 \mathrm{~min}$ at a flow rate of $20 \mu \mathrm{L} / \mathrm{min}$. Nitrogen was used as both nebulizing gas and drying gas at a flow rate of $10 \mathrm{~L} / \mathrm{h}$ for nebulization, and a flow of $200 \mathrm{~L} / \mathrm{h}$ for drying. Maximizing the flow of the nebulizing gas is important for optimum spraying. A low nebulizing rate results in accumulation of a tiny amount of the injected sample on the bottom of the probe tip, causing carryover and decreased sensitivity problems. The mobile phase used for most experiments was acetonitrile/water $(65: 35 \mathrm{vol} / \mathrm{vol})$ at a flow rate of 20 $\mu \mathrm{L} /$ min without splitting. The autosampler was programmed to inject a volume of $22 \mu \mathrm{L}$ of the sample every $3 \mathrm{~min}$, with one flush cycle of the mobile phase before and after each injection.

Quantitative analysis of acylcarnitines and amino acids. Calibration curves were established for three acylcarnitines (acetylcarnitine, $\mathrm{C}_{2}$; octanoylcarnitine, $\mathrm{C}_{8}$; and palmitoylcarnitine, $\mathrm{C}_{16}$ ), and also for amino acids; alanine (Ala), leucine (Leu), and phenylalanine (Phe) in control blood. A volume of $150 \mu \mathrm{L}$ of blood was spotted on filter paper and allowed to dry. This whole spot was taken in a tube and extracted as before with $7.5 \mu \mathrm{L}$ of methanol. Aliquots of this extract $(100 \mu \mathrm{L}$; equivalent to $2 \mu \mathrm{L}$ of blood) were taken. To each of these extracts were added $500 \mu \mathrm{L}$ of the internal standards solution containing the deuterated acylcarnitines $\left(\mathrm{C}_{2}, 0.15 \mathrm{nmol} ; \mathrm{C}_{8}\right.$, $\left.0.030 \mathrm{nmol} ; \mathrm{C}_{16}, 0.0625 \mathrm{nmol}\right)$ and the deutrated amino acids (Ala, Leu, Phe; $1.25 \mathrm{nmol}$ of each). To the same extracts were also added increasing amounts of the unlabeled acylcarnitines and amino acids. The resulting solutions were vortexed and then evaporated to dryness. The residues were derivatized with butanolic $\mathrm{HCl}$, and the procedure completed as described above (under blood sample preparation). The final injection mixtures were analyzed by automated ESI-MS/MS.

To examine the intra- and inter-day variation of the method, aliquots of $300 \mu \mathrm{L}$ of blood from a known case of methylmalonic acidemia and a control blood sample were spotted on filter paper and allowed to dry. The whole spot in each case was extracted in $7.5 \mu \mathrm{L}$ of methanol containing the labeled internal standards as described above. These extracts were dried and treated with $3.0 \mu \mathrm{L}$ of butanolic $\mathrm{HCl}$, and the procedure completed as described above. The final dried residues were reconstituted in $3.0 \mu \mathrm{L}$ of acetonitrile/water $(4: 1)$. Eight injections of each sample were made on each of $d 2,4$, and 6. The ratios of measured absolute heights for each of acetylcarnitine, propionylcarnitine, palmitoylcarnitine, alanine, leucine, and phenylalanine, to their respective internal standard were calculated after MS/MS data processing, and the coefficient of variation intra-day and inter-day were determined.

\section{RESULTS AND DISCUSSION}

\section{Acylcarnitine Profiling}

The butyl esters of acylcarnitines are well suited for analysis by positive-ion ESI-MS since they already carry a positive charge and accordingly, no additives are needed in the mobile phase. As such, they are detected as their respective molecular ions. Upon CID, these butyl esters yield a common fragment ion at $\mathrm{m} / \mathrm{z} 85$ corresponding to ${ }^{+} \mathrm{CH}_{2}-\mathrm{CH}=\mathrm{CH}-\mathrm{COOH}$. This fragment results from the loss of elements of both $\left(\mathrm{CH}_{3}\right)_{3} \mathrm{~N}$ and $\mathrm{C}_{4} \mathrm{H}_{8}$ and the side chain acyl group as $\mathrm{RCOOH}$. The precursor ion spectra thereby obtained show the molecular ion species corresponding to the butylated acylcarnitines in the sample.

Fatty acid oxidation defects. The acylcarnitine profile of a control blood sample is shown in Figure 1A. The profile is essentially very similar to those produced by FAB-MS/MS (1-3). Acylcarnitine profiles from cases with fatty acid oxidation disorders are shown in Figure $1, B, C$, and $D$. The acylcarnitine profile shown in Figure $1 B$ was obtained from a 20 -d-old patient with GA type 2 . The profile showed general elevation of the $\mathrm{C}_{4}$ to $\mathrm{C}_{18}$ acylcarnitines. This diagnosis was supported by GC/MS urine organic acid analysis which showed significantly elevated dicarboxylic acids, including ethylmalonic, glutaric, adipic, and suberic acid. Isovalerylglycine and isobutrylglycine were also elevated (data not shown).

The acylcarnitine profile shown in Figure $1 C$ is for a case of medium-chain acyl-CoA dehydrogenase deficiency in an American child, from whom a blood spot on an original Guthrie card was kindly provided by Dr. Piero Rinaldo (Yale University). The profile showed significant elevations in the $\mathrm{C}_{6}, \mathrm{C}_{8: 1}, \mathrm{C}_{8}$, and $\mathrm{C}_{10: 1}$ species that are characteristic of this disorder. This diagnosis had been previously established by GC/MS urine analysis of acylglycines and by molecular analysis (7).

The acylcarnitine profile shown in Figure $1 D$ represents a case of either long-chain or very long-chain acyl-CoA dehydrogenase deficiency from a 2-mo-old Saudi patient. Initial tandem MS analysis showed elevation of the $C_{14: 1}$ and $C_{14: 2}$ acylcarnitine species. Further investigation of this patient was carried out by carnitine loading for $24 \mathrm{~h}$ that resulted in a significant increase in $\mathrm{C}_{14: 1}$ and $\mathrm{C}_{14: 2}$ in addition to the $\mathrm{C}_{12}$ species. However, fasting for $8 \mathrm{~h}$ resulted in an even more prominent increase in the same species (data in Fig. 1D). A very similar FAB-MS/MS acylcarnitine profile was published earlier by Millington et al. (3). Enzyme and molecular characterization of our patient is in progress.

Branched chain amino acid disorders. Abnormal profiles from cases affected with disorders of branched-chain amino acid catabolism are shown in Figure $2, A, B, C$, and $D$. The acylcarnitine profile in Figure $2 A$ is for a case of isovaleric acidemia. The profile showed a very prominent ion at $\mathrm{m} / \mathrm{z} 302$ corresponding to isovalerylcarnitine. This preliminary diagno- 

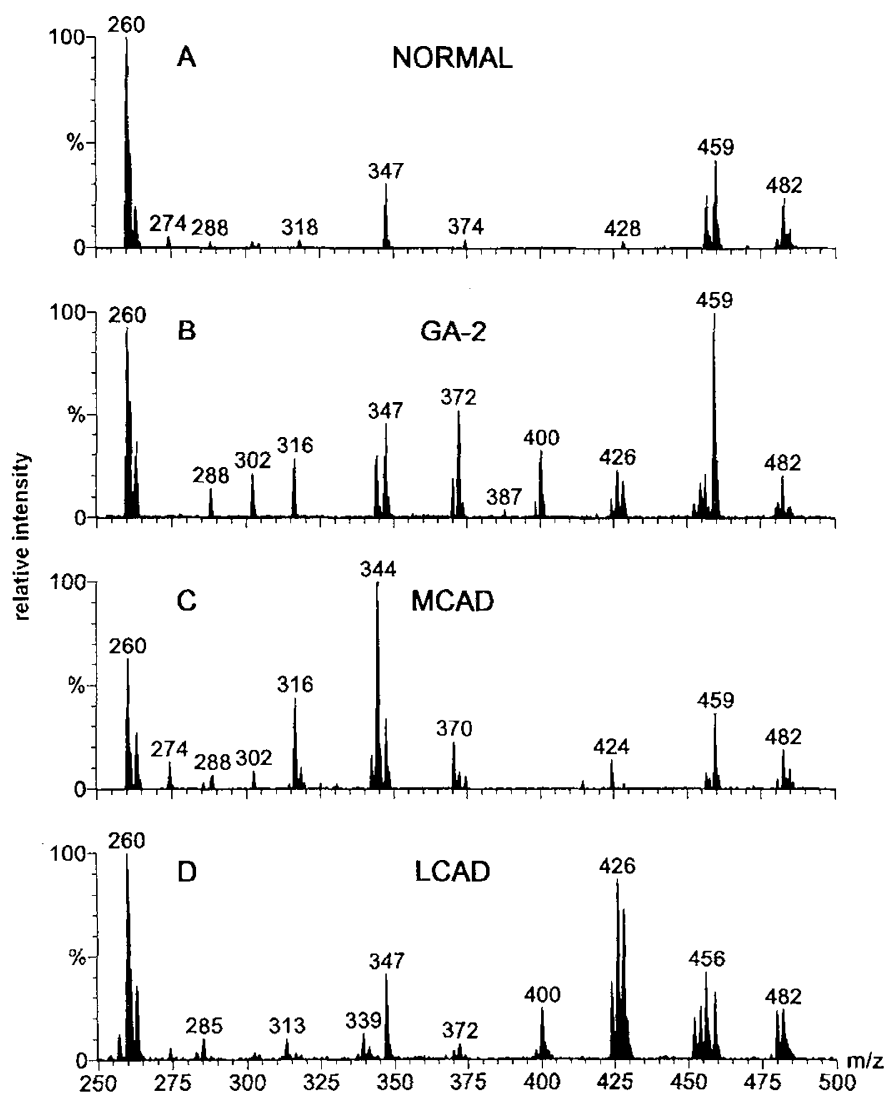

Figure 1. Blood acylcarnitine profiles obtained by ESI-MS/MS analysis using precursor-ion scanning of $\mathrm{m} / \mathrm{z} 85$. $(A)$ Normal; $(B)$ a case of GA type 2 $(G A-2) ;(C)$ a case of medium-chain acyl-CoA dehydrogenase deficiency $(M C A D) ;(D)$ a case of long chain acyl-CoA dehydrogenase deficiency $(L C A D)$. The signals in the profile are the molecular ions $\left(\mathrm{M}^{+}\right)$of the acylcarnitine butyl esters. Their masses are as follows: acetyl $\left(260 ; \mathrm{d}_{3}\right.$-labeled isotope 263), propionyl (274), butyryl or isobutyryl (288), isovaleryl or 2-methylbutryl (302), 2-methyl-3-hydroxybutyryl or 3-hydroxyisovaleryl (318), hexanoyl (316), octanoyl (344; $\mathrm{d}_{3}$-labeled isotope 347), decanoyl (372), glutaryl (388), dodecanoyl (400), 3-methylglutaryl (402), tetradecanoyl (428), hexadecanoyl (456; $\mathrm{d}_{3}$-labeled isotope 459), octadecanoyl (484). Their unsaturated analogs appear $2 \mathrm{D}$ lower in mass.

sis was further confirmed by GC/MS urine organic acid analysis (data not shown).

The acylcarnitine profile in Figure $2 B$ was obtained from a blood sample of a 1-y-old girl which was sent to our laboratory with no prior diagnosis. The acylcarnitine data suggested a possible diagnosis of $\beta$-ketothiolase deficiency due to the presence of prominent ions consistent with tiglylcarnitine $(\mathrm{m} / \mathrm{z}$ 300) and 2-methyl-3-hydroxybutyrylcarnitine (m/z 318). The GC/MS analysis of urine organic acids confirmed the MS/MS results by the finding of excessive amounts of 2-methyl-3hydroxybutyric acid (1793 $\mathrm{mmol} / \mathrm{mol}$ creatinine; normal range is 3-72) and tiglylglycine (not quantified).

The profile shown in Figure $2 C$ was obtained from a blood sample from a 1-mo-old Saudi girl. The acylcarnitine data suggested a possible diagnosis of HMG. The spectrum showed an ion at $\mathrm{m} / \mathrm{z} 402$ consistent with 3-methylglutarylcarnitine and a more prominent ion at $\mathrm{m} / \mathrm{z} 318$ presumably corresponding to 3-hydroxyisovalerylcarnitine. The ion at $\mathrm{m} / \mathrm{z} 402$, although not abundant, is strongly suggestive of $\mathrm{HMG}$, because it has not been observed in blood of normal patients, or in other organic
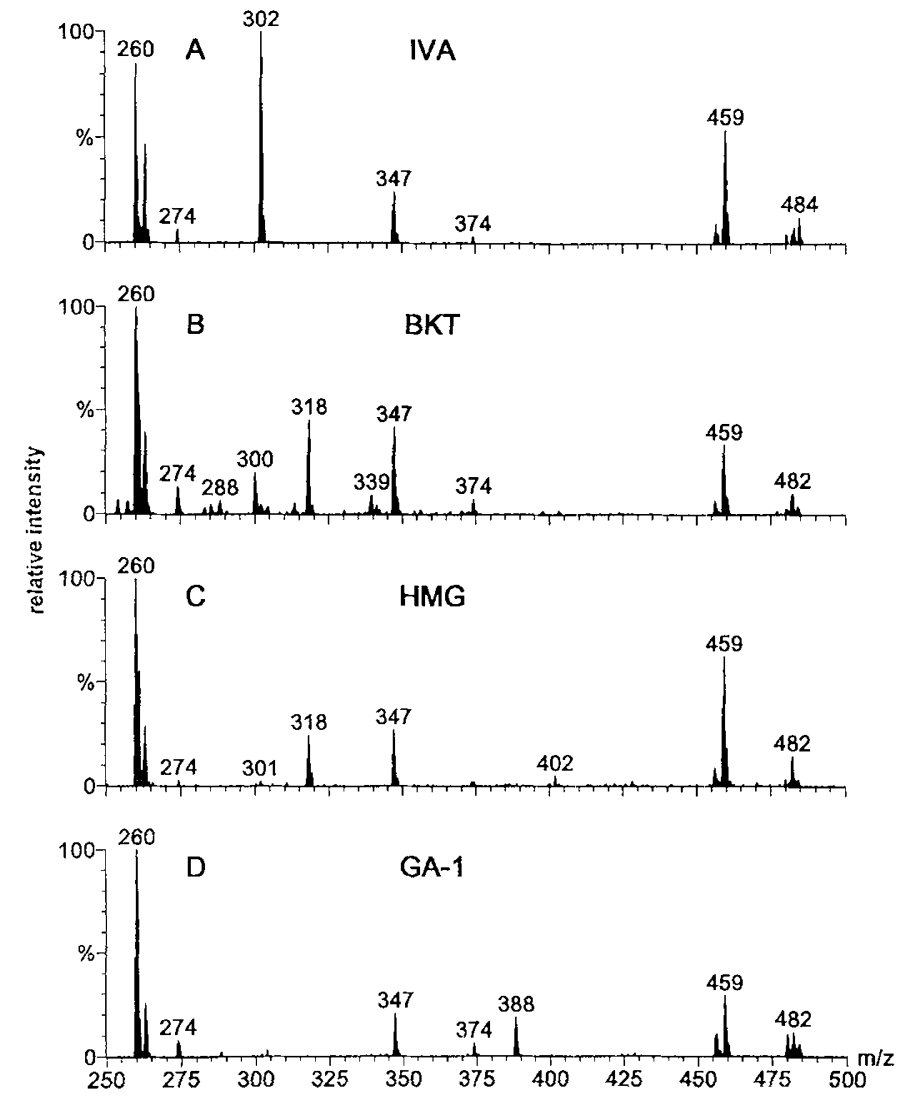

Figure 2. Blood acylcarnitine profiles obtained by ESI-MS/MS analysis, scanning for precursor ions of $\mathrm{m} / \mathrm{z} 85$. (A) A case of isovaleric acidemia (IVA); $(B)$ a case of $\beta$-ketothiolase deficiency $(B K T) ;(C)$ a case of $\mathrm{HMG} ;(D)$ a case of GA type 1 $(G A-l)$. A key for the masses is provided in the legend for Figure 1.

acidemia cases diagnosed by this technique (1). In four cases, HMG was diagnosed by tandem MS and was later confirmed by GC/MS urine organic acid analysis and enzyme assay in fibroblasts as previously described $(8,9)$.

Figure $2 D$ shows a typical acylcarnitine profile observed in patients with GA type 1 . The spectrum was apparently normal except for the appearance of an ion at $\mathrm{m} / \mathrm{z} 388$ corresponding to glutarylcarnitine. It is this ion which suggests GA type 1, as it is not normally observed, except in cases of GA type 2, where several additional ions are elevated (see above). These data were supported by GC/MS analysis of urine, which showed abnormal amounts of glutaric acid $(2685 \mathrm{mmol} / \mathrm{mol}$ creatinine; normal, $<2)$ as well as 3-hydroxyglutaric acid (211 $\mathrm{mmol} / \mathrm{mol}$ creatinine; normal, $0-3)(8)$.

\section{Amino Acid Profiling}

ESI-MS/MS was applied to the analysis of amino acids after extraction from blood spots and derivatization to butyl esters. The butylated derivatives fall into two groups. With the exception of six of the amino acids (these being asparagine, glutamine, arginine, citrulline, lysine, and ornithine), the butylated amino acid species detected by this technique give a common "neutral loss" of 102 D, upon CID fragmentation. This corresponds to the loss of butylformate from their protonated molecular ions. It is noteworthy to mention that glycine 
produces a very weak signal and can be easily missed. The above mentioned six amino acids lose ammonia first, followed by butylformate, resulting in a total neutral loss of $119 \mathrm{D}$. Therefore, to get a more complete picture of the amino acids in the sample, two constant neutral loss scan functions were used, scanning for losses of 102 and $119 \mathrm{D}$, generating a separate profile for each scan function.

Amino acid disorders. A typical normal amino acid profile obtained by scanning for a neutral loss of $102 \mathrm{D}$ is shown in Figure $3 A$. Abnormal profiles from cases affected with amino acid catabolism disorders are shown in Figures 3 and 4. The amino acid profile shown in Figure $3 B$ is characterized by a very prominent ion at $\mathrm{m} / \mathrm{z} 222$ corresponding to protonated molecular ion of butylated phenylalanine, and a very high ratio of phenylalanine to tyrosine $\left(\mathrm{MH}^{+}=238\right)$, estimated to be about 18 . These results were clearly consistent with a diagnosis of PKU (4).

The profile in Figure $3 C$ showed a very prominent ion at $\mathrm{m} / \mathrm{z}$ 188 corresponding to leucine/isoleucine, a finding suggestive
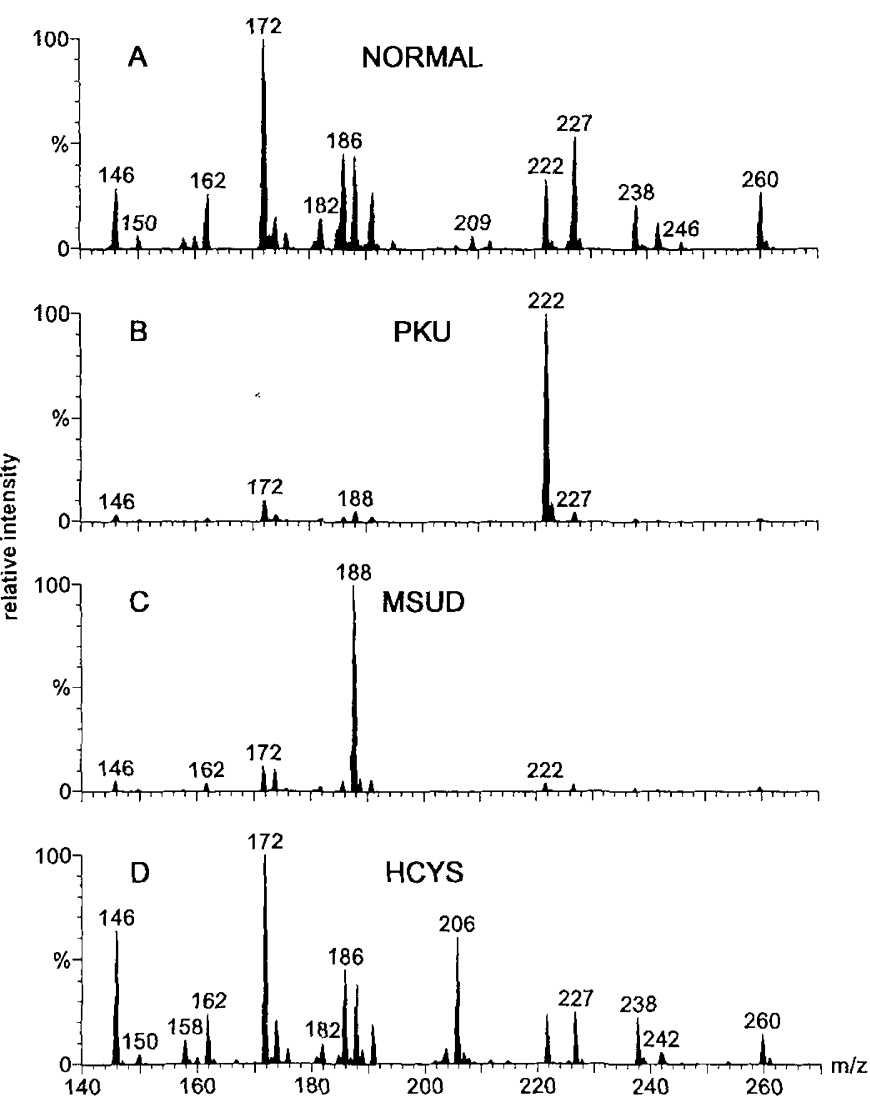

Figure 3. Blood amino acid profiles obtained by ESI-MS/MS analysis, scanning for a constant neutral loss of $102 \mathrm{D}$. $(A)$ Normal; $(B)$ a case of PKU; $(C)$ a case of maple syrup urine disease $(M S U D) ;(D)$ a case of homocystinuria (HCYS). The signals in the profile correspond to the protonated molecular ions $\left(\mathrm{MH}^{+}\right)$of amino acid butyl esters. Their masses are as follows: alanine (146; $\mathrm{d}_{4}$-labeled isotope 150$)$, serine (162), proline (172), valine (174; $\mathrm{d}_{8}$-labeled isotope 182), threonine (176), pyroglutamic/deammoniated glutamine and lysine/pipecolic acid (186), leucine/isoleucine (188; $\mathrm{d}_{3}$-labeled isotope 191), asparagine/ornithine (189), glutamine/lysine (203), methionine (206; $\mathrm{d}_{3}$. labeled isotope 209), histidine (2I2), phenylalanine (222; $\mathrm{d}_{5}$-labeled isotope 227 ), arginine $(231)$, citrulline (232), tyrosine (238; $\mathrm{d}_{4}$-labeled isotope 242 ), aspartate dibutyl ester (246), tryptophan (261), and glutamate dibutyl ester $(260)$.
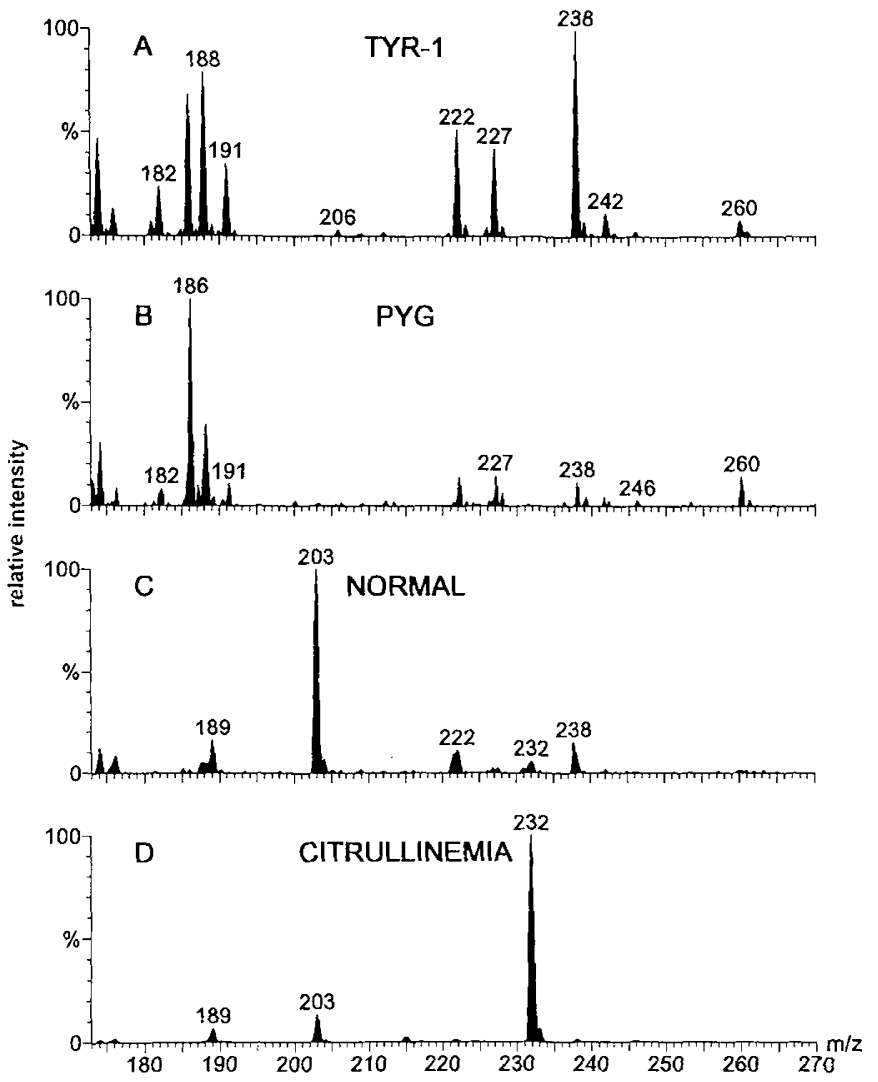

Figure 4. Blood amino acid profiles obtained by ESI-MS/MS analysis. Neutral loss scanning of $102 \mathrm{D}(A$ and $B)$. (A) A case of tyrosinemia tpye-1 $(T Y R-1) ;(B)$ a case of pyroglutamic acidemia $(P Y G)$. Neutral loss scanning of $119 \mathrm{D}(C$ and $D)$. $(C)$ Normal; $(B)$ a case of citrullinemia. A key for the masses is provided in the legend for Figure 3.

of maple syrup urine disease. The profile in Figure $3 D$ was obtained from the blood sample of a 6-y-old female Saudi patient previously diagnosed as suffering from homocystinuria due to cystathionine $\beta$-synthetase deficiency. The enzyme assay showed $<1 \%$ of normal activity. Accordingly, the amino acid profile showed a very prominent ion at $\mathrm{m} / \mathrm{z} 206$ corresponding to the butyl derivative of methionine, an amino acid that is normally very low or undetectable by this method in normal blood.

Figure $4 A$ shows an abnormal profile from the blood sample of a Saudi girl clinically suspected to have tyrosinemia type 1 . The profile showed an abundant ion at $\mathrm{m} / \mathrm{z} 238$ corresponding to the butyl derivative of tyrosine, which agreed with the clinical observations. These data were further supported by the detection of a significant elevation of succinylacetone in the patient's urine by GC/MS analysis. A very similar profile was obtained from the blood sample of a 28-y-old Saudi man, previously diagnosed clinically and biochemically (plasma HPLC for amino acids) as suffering from tyrosinemia type 2. Obviously, additional investigations, using other methods, are needed to distinguish the two types of tyrosinemia and whether either of these disorders is the true underlying cause for the elevation in tyrosine level.

In Figure $4 B$ the prominence of the ion at $\mathrm{m} / \mathrm{z} 186$ that corresponds to the butyl derivative of pyroglutamic acid (5oxoproline) suggested a possible diagnosis of pyroglutamic 
acidemia due to glutathione synthetase deficiency. However, ions with the same mass are produced from the deammoniated $\left(\mathrm{MH}^{+}-\mathrm{NH}_{3}\right)$ fragment of the butyl derivative of both glutamine and lysine $\left(\mathrm{MH}^{+}=203\right)$, as well as from the protonated molecular ion of the imino acid, pipecolic acid $\left(\mathrm{MH}^{+}=\right.$ 186). This situation makes confirmation by other techniques essential. For this reason we requested a urine sample from the patient for GC/MS analysis of organic acids. The urine profile showed a massive amount of pyroglutamic acid, fully consistent with the MS/MS results. To investigate the utility of the technique in screening for this particular disorder, we recalled two other previously diagnosed cases of pyroglutamic acidemia, where the diagnosis was based on GC/MS analysis of urine. ESI-MS/MS of blood samples from these patients also showed significant elevation of the ion at $\mathrm{m} / \mathrm{z} 186$ (data not shown), providing additional evidence that ESI-MS/MS can be used to screen for this disease.

Urea cycle disorders. A normal amino acid profile obtained by neutral loss scanning of $119 \mathrm{D}$ is shown in Figure $4 \mathrm{C}$. Typically, the spectrum showed a major ion at $\mathrm{m} / \mathrm{z} 203$ corresponding to the protonated molecular ions of the butyl derivatives of both glutamine and lysine. Minor contributions from valine, threonine, asparagine, ornithine, phenylalanine, citrulline, and tyrosine were also observed in the spectrum.

Figure $4 D$ shows an abnormal profile for a patient suspected of having a urea cycle disorder. The profile of a neutral loss of $119 \mathrm{D}$ showed an abundant ion at $\mathrm{m} / \mathrm{z} 232$ corresponding to the butyl derivative of citrulline. The level of citrulline in this case is at least 50 -fold higher than in the normal sample, a finding suggestive of citrullinemia. In addition to the ion at $\mathrm{m} / \mathrm{z} 232$, there is also an ion at $\mathrm{m} / \mathrm{z} 215$ which corresponds to the deammoniated butyl derivative of citrulline. The ion at $\mathrm{m} / \mathrm{z} 215$ subsequently loses butylformate and therefore appears in the neutral loss scan function of $102 \mathrm{D}$.

\section{Automation of Sample Introduction}

One of the main objectives of this work was the automation of sample introduction into the mass spectrometer, to increase the sample throughput. We found that acceptable acylcarnitine profiles can be obtained by acquiring about 20 scans for the precursor ions of $\mathrm{m} / \mathrm{z} 85$. Amino acid profiles of an equally acceptable signal-to-noise quality are obtained from 10 scans for each of the neutral loss functions. The total number of 40 scans thus required needs about $90 \mathrm{~s}$ of sample presence in the ion source.

Figure $5 \mathrm{~A}$ shows a total ion chromatogram of 15 consecutive injections of a standard solution containing the butyl ester derivatives of three stable isotope-labeled acylcarnitines $\left(\mathrm{C}_{2}\right.$, $\mathrm{C}_{8}$, and $\mathrm{C}_{16}$ ). Precursor ion scans of $\mathrm{m} / \mathrm{z} 85$ were acquired in the MCB mode under the above mentioned conditions (see Methods). A flow rate of $20 \mu \mathrm{L} / \mathrm{min}$ was used, giving peak widths (measured at baseline) of approximately $1.7 \mathrm{~min}$ or 102 $s$ which was quite adequate for the number of scans needed. This gave a 1-min separation between the trailing and leading sides of successive peaks. As can be observed from Figure $5 \mathrm{~A}$, the signal essentially falls to background level between peaks, which is important to assure the lack of interference between
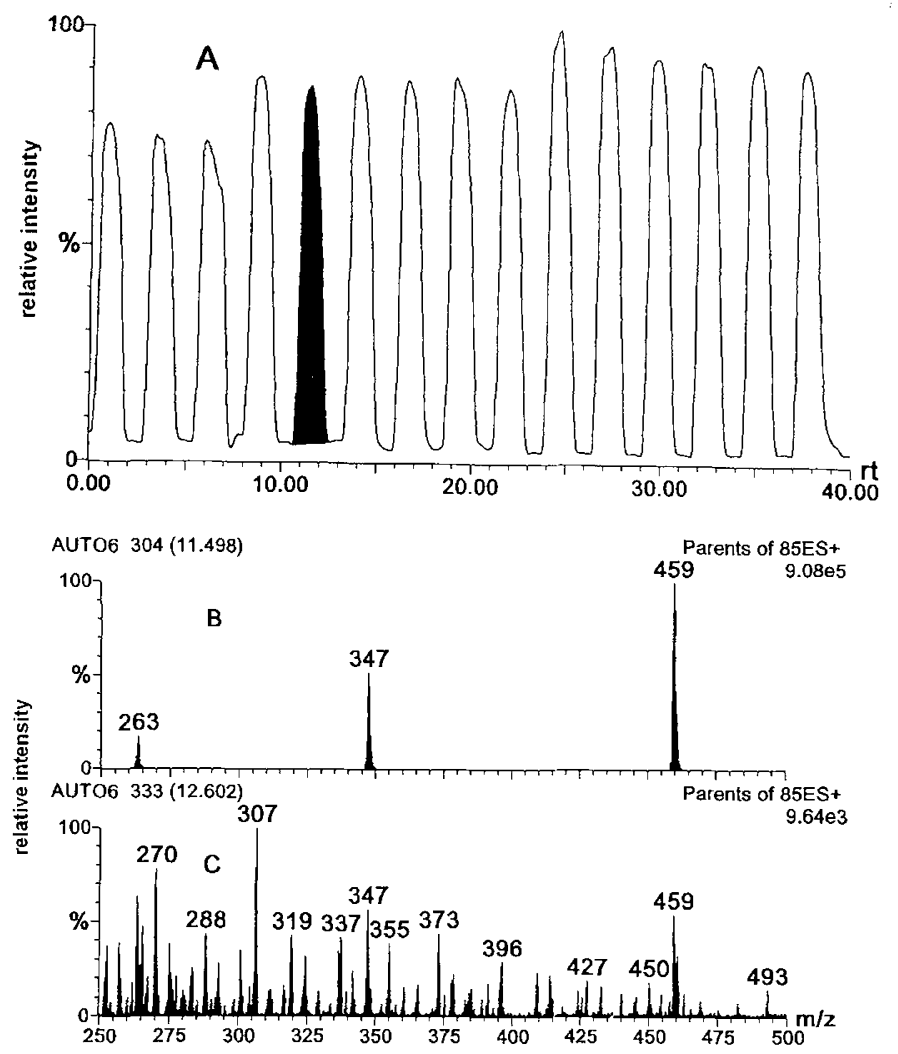

Figure 5. Automated ESI-MS/MS analysis of standard acylcarnitine solution acquired in the continuum mode (MCB), scanning for the precursor ions of $\mathrm{m} / \mathrm{z}$ 85. (A) Total ion chromatogram obtained from 15 consecutive injections of a standard solution containing three stable isotope labeled acylcarnitine butyl esters $\left(C_{2}=\mathrm{m} / \mathrm{z} 263, C_{8}=\mathrm{m} / \mathrm{z} 347\right.$, and $\left.C_{16}=\mathrm{m} / \mathrm{z} 459\right)$. (B) Averaged spcctrum of the highlighted peak in $A$. (C) Averaged spectrum for 15 consecutive scans at end of the highlighted peak in $A$.

successive samples in the sequence. Figure $5 B$ shows the averaged spectrum for the peak 5 (shaded) eluting at about 11.5 min, whereas Figure $5 \mathrm{C}$ shows the averaged spectrum for 15 scans in the region after the peak. A comparison of the two spectra clearly indicated that the level of the acylcarnitines fell to background level between peaks.

To test the possibility of sample carryover or interference from one sample to the next, 22 consecutive injections were made. The first and last injections were blank extracts containing only the internal standards, the remaining 20 injections were all patient samples. Using the $\mathrm{MCB}$ acquisition mode, the mass spectrometer was programmed to repeatedly cycle through three scan functions as follows: five scans for the precursors of $\mathrm{m} / \mathrm{z} 85$, three scans for neutral loss of $102 \mathrm{D}$, followed by three scans for neutral loss $119 \mathrm{D}$. The total ion chromatogram obtained for the precursor ions of $\mathrm{m} / \mathrm{z} 85$ (acylcarnitines) is shown in Figure 6. Actually, 15 of the 20 patient samples were from known abnormal cases. Two examples are given to demonstrate the absence of carryover between samples. Figure $7, A$ and $B$, shows the acylcarnitine profiles obtained by averaging the spectra for each of peaks 8 and 9 (of Fig. 6), respectively. The spectrum in Figure $7 A$ is for a known case of GA type 2, whereas that in Figure $7 B$ is for a control blood sample. In the same way, Figure $7, C$ and $D$, shows the amino acid profiles corresponding to each of peaks 12 and 13 , 


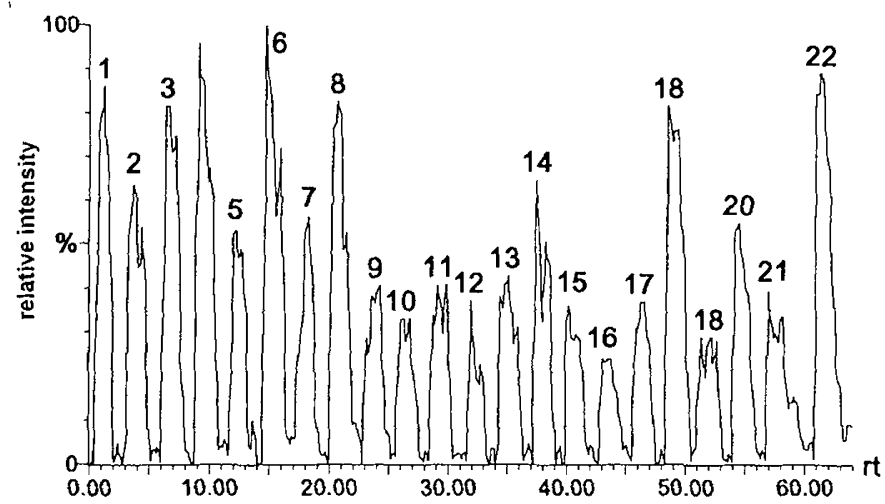

Figure 6. Total ion chromatogram obtained by automated ESI-MS/MS analysis of patient samples acquired in the continuum mode (MCB). Twenty-two consecutive injections were made, 20 of which were patient samples, whereas the first and the last peaks are for blank extracts of only internal standards. The data were acquired by cycling between three scan functions: five scans of precursor-ions of $\mathrm{m} / \mathrm{z} 85$, three scans for neutral loss of $102 \mathrm{D}$, followed by three scans for neutral loss of $119 \mathrm{D}$. The width of the peak was about $1.7 \mathrm{~min}$, measured at baseline.

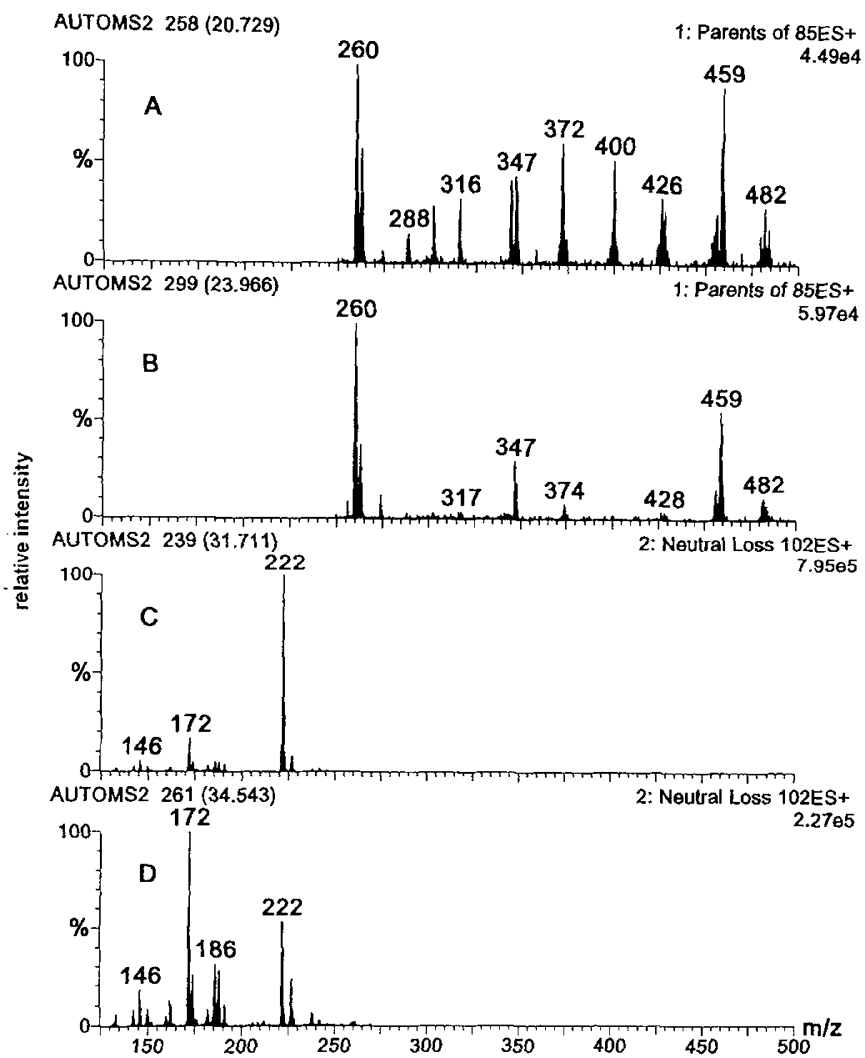

Figure 7. Comparison of two sets of mass spectra obtained from consecutive automatic injections (data from Fig. 6). (A) Averaged spectrum for precursor ions of $\mathrm{m} / \mathrm{z} 85$ from peak 8 (a case of GA type 2 ); $(B)$ averaged spectrum for precursor ions of $\mathrm{m} / \mathrm{z} 85$ for peak 9 (control blood); $(C)$ averaged spectrum for neutral loss of $102 \mathrm{D}$ (a case of PKU); $(D)$ averaged spectrum for neutral loss of $102 \mathrm{D}$ (control blood)

respectively. The first is for a known case of PKU, whereas the latter is for a control blood sample. It is clearly apparent in both examples that there is no carryover from one peak to the next. The same absence of carryover was observed for all the other abnormal samples injected in this sequence.
To date, the largest number of samples injected in one automatic sequence was 200 samples, over a 10 -h period. Theoretically, 480 samples can be analyzed in a 24 -h period; however, further work is needed to determine whether the system can actually handle such a large sample throughput every day without loss of sensitivity and to determine the appropriate source cleaning intervals.

\section{Quantification and Reproducibility}

In the initial development work, no quantification was carried out. The addition of the internal standards to the blood sample served as an indicator for recovery, and a standard solution containing these species was used to tune the mass spectrometer. In a latter stage of this study, determinations were carried out to examine the quantitative merits of the technique and its reproducibility. Calibration curves were linear in the range $0.5-50 \mathrm{nmol} / \mu \mathrm{L}$ of blood for acetylcarnitine $\left(r^{2}=0.996\right)$, for octanoylcarnitine $\left(r^{2}=0.995\right)$ and for palmitoylcarnitine $\left(r^{2}=0.996\right)$. The calibration curves were linear for alanine $\left(r^{2}=0.992\right.$, range of $5-500 \mathrm{nmol} / \mu \mathrm{L}$ of blood), leucine $\left(r^{2}=0.996\right.$; range of $\left.5-500 \mathrm{nmol} / \mu \mathrm{L}\right)$, and for phenylalanine $\left(r^{2}=0.997\right.$; range of $\left.5-800 \mathrm{nmol} / \mu \mathrm{L}\right)$. These results demonstrate the utility of the technique in quantifying both acylcarnitines and amino acids at levels well below normal, as well as for significantly elevated levels. Therefore, the method can clearly distinguish between normal and abnormal cases.

The precision of the assay was examined by repeated injections $(n=8)$ of one abnormal case (methylmalonic acidemia) and one normal sample. The results were excellent except for one of six analytes. Coefficients of variation percent were as follows (intra-day; inter-day): For the methylmalonic acidemia sample, acetylcarnitine $(2.51 ; 3.02)$, propionylcarnitine $(3.81$; $3.81)$, palmitoylcarnitine $(5.11 ; 5.57)$, alanine $(22.03 ; 24.76)$, leucine $(6.90 ; 7.12)$, and phenylalanine $(4.11 ; 5.08)$. The results for the normal sample were very much the same or better than for the abnormal sample (data not shown). We feel that the high variation for alanine is due to the poor signal response for this compound and its deutrated analog, on an equimolar basis, relative to that for leucine and phenylalanine. In consequence, the signal to noise ratio for the amount of deuterated alanine currently added, is too low to give consistent ratio measurements. This can possibly be corrected by increasing the concentration of deutrated alanine in the internal standard mixture. A study is underway to determine the effects of long-term storage of spotted blood samples on the quality of the data.

In summary, the ESI-MS/MS system proved to be highly reliable. There was not a single breakdown in 9 mo of operation, and the ESI ion source was cleaned only once a month; a process that takes less than $30 \mathrm{~min}$. The method described was found to be specific, accurate, and can easily discern normal from abnormal results. The profiles for extracted samples showed excellent reproducibility when samples were repeatedly analyzed on the same day, or at a latter time.

ESI-MS/MS is proven by our studies to be a primary tool for the diagnosis of about 20 different organic acid and amino acid 
catabolism disorders. In the last 9 mo we have analyzed approximately 2000 blood samples, diagnosed 52 new cases, and confirmed the diagnosis of another 75 previously known cases. The relatively large number of cases found in the last 9 mo reflects the high incidence of organic acid and amino acid disorders in Saudi Arabia, which is probably due to the relatively high level of consanguinity, because most of the diseases cluster in certain tribes (13). The most common diseases detected in this study were methylmalonic acidemia, propionic acidemia, PKU, maple syrup urine disease, $\mathrm{HMG}$, GA type 1, and $\beta$-ketothiolase deficiency, in decreasing order. We believe that the successful automation of sample introduction achieved here should bring closer the possibility for developing "broad spectrum" neonatal screening programs for inborn errors of metabolism.

Acknowledgments. The authors express our deepest gratitude to Dr. Abbass Al Marzouky, Executive Director, and Dr. Sultan Al Sedairy, Deputy Executive Director, of the Research Centre of the King Faisal Specialist Hospital and Research Centre, Riyadh, for their continued support, without which this work would not have been possible. We thank Dr. David S. Millington of Duke University for advice and materials. We also thank our colleagues, Dr. Subramanyam Sarvepalli, Mohamed Al-Amoudi, Nazih Youssef, Ali Al-Odaib, Amin Awad, and Minnie Jacob for their technical assistance. Finally, we thank Maria Theresa Pablo for her typing and secretarial efforts.

\section{REFERENCES}

1. Millington DS, Kodo N, Norwood DL, Roe CR 1990 Tandem mass spectrometry: A new method for acylcarnitine profiling with potential for neonatal screening for inborn errors of metabolism. J Inherit Metab Dis 13:321-324

2. Millington DS, Kodo N, Terada N, Roe D, Chace DH 1991 The analysis of diagnostic markers of genetic disorders in human blood and urine using tandem mass spectrometry with liquid secondary ion mass spectrometry. Int J Mass Spectrom Ion Processes 111:211-228

3. Millington DS, Terada N, Chace DH, Chen Y, Ding J, Kodo N, Roe CR 1992 The role of tandem mass spectrometry in the diagnosis of fatty acids oxidation defects. In: Coates PM, Tanaka K (eds) New Developments in Fatty Acid Oxidation. Wiley-Liss, New York, pp 339-354

4. Chace DH, Millington DS, Terada N, Kahler SG, Roe CR, Hofman LF 1993 Rapid diagnosis of phenylketonuria by quantitative analysis of phenylalanine and tyrosine in neonatal blood spots by tandem mass spectrometry. Clin Chem 38:1-6

5. Kelly BM, Rose ME, Wycherley D, Preece, SW 1992 Electrospray mass spectra of medium-chain and long-chain acylcarnitines. Org Mass Spectrom 27:924-926

6. Rashed MS, Ozand PT, Harrison ME, Watkins PJF, Evans S 1994 Electrospray tandem mass spectrometry in the diagnosis of organic acidemias. Rapid Commun Mass Spectrom 8:129-133

7. Rinaldo P, O'Shea JJ, Coates PM, Hale DE, Stanley CA, Tanaka K 1988 Mediumchain acyl-CoA dehydrogenase deficiency. Diagnosis by stable-isotope dilution measurement of urinary $n$-hexanoylglycine and 3-phenylpropionylglycine. N Engl J Med 319:1308-1313

8. Sweetman L 1991 Organic acid analysis. In: Hommes A (ed) Techniques in Diagnostic Human Biochemical Genetics: A Laboratory Manual. Wiley-Liss, New York, pp 143-176

9. Ozand PT, Al-Aqeel A, Gascon G, Brismar J, Thomas E, Gleispach H 1991 3-Hydroxy-3-methylglutaryl-coenzyme A (HMG-CoA) lyase deficiency in Saudi Arabia. J Inherit Metab Dis 14:174-188

10. Rashed MS, Ozand PT, Al-Aqeel, Gascon G 1994 Experience of King Faisal Specialist Hospital and Research Center with Saudi organic acid disorders. Brain Dev 16:(suppl): $1-6$ 